\title{
Sub-20-K Noise Temperature LNA for 67-90 GHz Frequency Band
}

\author{
Pekka Kangaslahti ${ }^{1}$, Kieran Cleary ${ }^{2}$, Jacob Kooi ${ }^{1}$, Lorene Samoska ${ }^{1}$, Richard Lai ${ }^{3}$, Michael Barsky ${ }^{3}$, Xiaobing Mei $^{3}$, \\ Stephen Sarkozy ${ }^{3}$, and Mikko Varonen ${ }^{4}$ \\ ${ }^{1}$ Jet Propulsion Laboratory, California Institute of Technology, Pasadena, CA 91109 USA \\ ${ }^{2}$ California Institute of Technology, Pasadena, CA 91109 USA \\ ${ }^{3}$ Northrop Grumman Corporation, Redondo Beach, CA 91109 USA \\ ${ }^{4}$ Aalto University, Espoo, Finland
}

\begin{abstract}
Indium Phosphide MMIC LNAs are enabling new capabilities in instrument development. The development of arrays of hundreds of cryogenically-cooled millimeter wave receivers has previously been challenging, but is now achievable with highly repeatable MMIC processes and advances in cryogenic on-wafer testing of LNAs. We have developed InP HEMT LNA MMICs for the 67-90 GHz frequency band that is the last missing receiver system from the ALMA. These MMICs provided average performance of less than $22.5 \mathrm{~K}$ noise temperature over the frequency band and minimum noise temperature of $17.5 \mathrm{~K}$ at $72 \mathrm{GHz}$. These LNAs achieve $\mathrm{NT}=220 \mathrm{~K}(\mathrm{NF}=2.4 \mathrm{~dB})$ at $90 \mathrm{GHz}$ for Earth remote sensing instrument on Sentinel-6. Our HRMR (High Resolution Microwave Radiometer) achieves NEDT < 0.05K enabling Sentinel-6 to measure coastal ocean topography at $3 \mathrm{~km}$ resolution with better than $1 \mathrm{~cm}$ accuracy.

Index Terms- MMIC, LNA, InP, ALMA, cryogenic, ocean topography, Sentinel-6
\end{abstract}

\section{INTRODUCTION}

Receiver arrays are attractive for enhancing the sensitivity of a radiometer by scanning multiple pixels simultaneously, or for synthesizing large apertures by using interferometry. These receiver arrays are easiest to build using MMICs due to the repeatability of the circuits and the ability to prescreen the MMICs before assembly. The largest astronomical instrument in operation is the Atacama Large Millimeter and submillimeter Array (ALMA) in northern Chile at an elevation of $5 \mathrm{~km}$. ALMA consists of 54 12-meter antennas and 12 7-meter antennas; each antenna system includes a cryostat with space for receiver cartridges in ten different bands. The only remaining receiver system that was not yet installed or in production is band 2 covering 67 to $90 \mathrm{GHz}$ in two polarizations.

Recent advances in InP technology have resulted in amplifiers with minimum noise temperatures in the 22 to $34 \mathrm{~K}$ range in this frequency range [1]-[4]. We designed InP MMICs in $35 \mathrm{~nm}$ gate length technology for this frequency band. These LNAs achieved $17.5 \mathrm{~K}$ noise temperature at 72 $\mathrm{GHz}$ and an average of $22.5 \mathrm{~K}$ of noise temperature over the full frequency band. These are the lowest noise temperatures reported at this frequency band for a cryogenically cooled packaged amplifier.

The InP technology is well suited for both cryogenically cooled and room temperature operation. We applied these LNAs for $90 \mathrm{GHz}$ receivers on Sentinel-6 ocean topography mission. Sentinel-6 carries a radar altimeter to provide high precision and timely observations of the topography of the global ocean. This information is essential for the continued monitoring of changes in sea level, and for operational oceanography. The path delay of the radar altimeter signal varies depending on the quantity of water vapor in the atmosphere. To achieve under one centimeter accuracy in the ocean topography measurement the water vapor is characterized with microwave radiometers. For extending this calibration within a few kilometers of the coast our HRMR instrument uses millimeter wave frequency receivers to improve the spatial resolution of the water vapor measurement with the available one meter antenna aperture [5]. These $35 \mathrm{~nm}$ LNAs provide the required low noise for direct detection radiometers that operate with low power and fit in the low volume available on the focal plane of the reflector.

\section{DESIGNS OF LNAS}

Indium Phosphide HEMT technology has advanced from $100 \mathrm{~nm}$ technology to under $35 \mathrm{~nm}$ gate lengths [4],[6],[7]. As expected, with the reduction in gate length, the transconductance of our devices improved from close to $1000 \mathrm{mS} / \mathrm{mm}$ to $2300 \mathrm{mS} / \mathrm{mm}$ and beyond. The gate capacitance was also reduced and this led to high $F_{t}$ of above $400 \mathrm{GHz}$. Further improvements in contact resistances and transistor design reduced the noise of the device. A significant factor for producing low noise amplifiers is the sharp turn-on of these devices that leads to high transconductance at the low bias currents that are required for low noise performance. With these optimum device characteristics available in our InP technology, the remaining task is to decide on the number of fingers and finger width of the device to use in the design of the low noise amplifier. At W-band we demonstrated that a four finger device with 15 um fingers would be optimum for a cryogenic LNA [8].

\section{A. Implementation of LNA Design}

After the selection of device size a few different LNA design topologies were used for the final low noise amplifiers to achieve variation in the processed LNAs. Both three-stage and two-stage common-source designs were implemented with microstrip matching circuits on the 2-mil thick substrate. Standard MIM thin-film capacitor and TaN resistor processes 
were available to design the bias circuits on the LNA MMIC. Fig. 1 and Fig. 2 show photos of the designed MMICs.

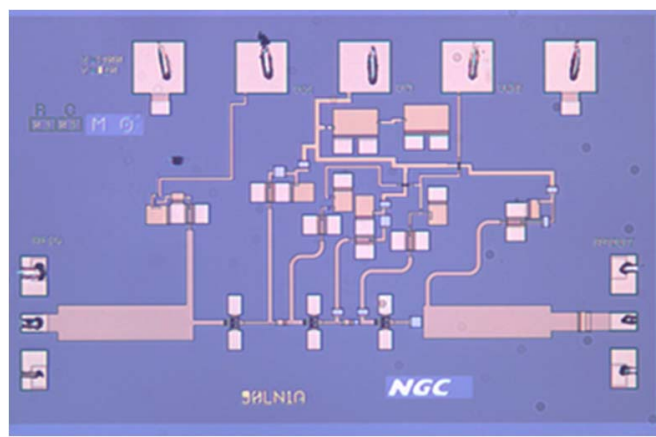

Fig. 1. A three-stage common-source design. The size of the MMIC is 1300 um x 820 um

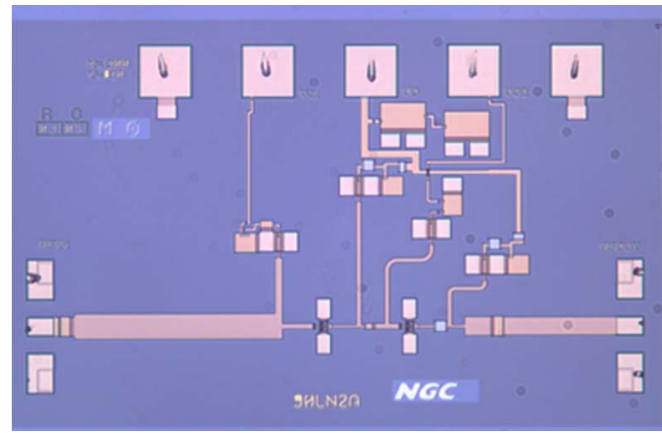

Fig. 2. A two-stage common-source design. The size of the MMIC is 1300 um x 820 um.

\section{B. LNA Processing}

The processing of the layouts included four three-inch wafers, two each of $75 \%$ and $100 \%$ Indium content in the device channels. The $75 \%$ channel results in slightly lower maximum transconductance, and provides a variation in the transistor performance which can improve the likelihood of success for the LNA design. The wafers contained several different designs of various operating frequencies, so the quantities of chips per design were 25-90 per wafer. The four wafers were tested at room temperature after thinning the wafers to $50 \mathrm{um}$ and then the wafers were diced for cryogenic testing. The onwafer yield was in the $85-90 \%$ range.

\section{LNA TESTING}

The on-wafer testing of LNAs allowed us to pick the amplifier chips that functioned well at room temperature. However, we have not been able to predict which of the LNAs achieve best cryogenic noise performance based on this room temperature data. We demonstrated that with a purpose built cryogenic test station the diced MMICs can be screened for low noise performance [9],[10]. Fig. 3. shows cryogenic test data for onchip testing of our two-stage LNA MMICs and Fig. 4 includes the data for the three-stage LNA. The probe station provides a relative, not absolute, measurement due to uncertainties in the temperature of the lossy part of the input probe. However, a relative measurement allows for selection of best performing chips and designs. This is highly valuable for cryogenic array development as the best cryogenic LNAs can be selected for use as the first amplifiers in the receiver.

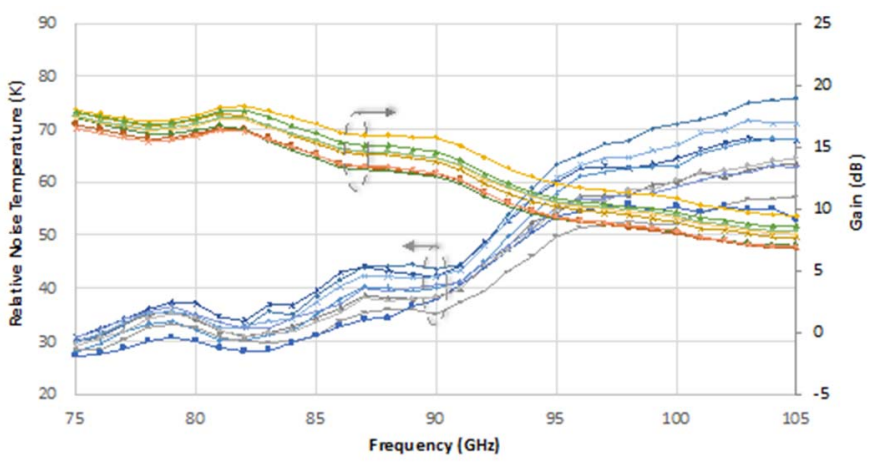

Fig. 3. Gain and relative noise temperature of screened 2-stage LNA MMICs. These on-chip measured data are for nine different chips of the same twostage design in Fig. 2. All chips show good performance, but the best ones can be selected for first stages of the cryogenic receivers.

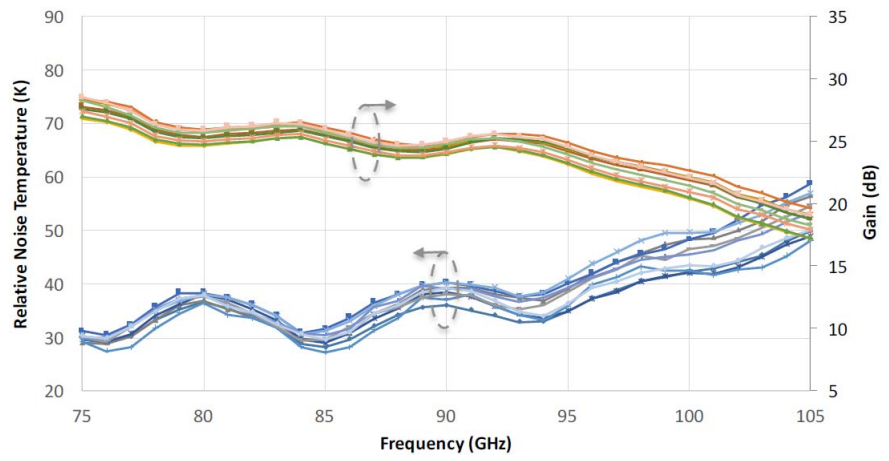

Fig. 4. Gain and relative noise temperature of 3-stage LNA MMICs

We picked the screened lowest-noise amplifiers for packaging in split-block brass housings with thin-film microstrip to waveguide transitions (Fig. 5). These housings had WR-10 waveguides that have cut-off at $58 \mathrm{GHz}$ and normal operating range from 75-110 GHz. This may degrade the presented result at the lower end of the frequency band.

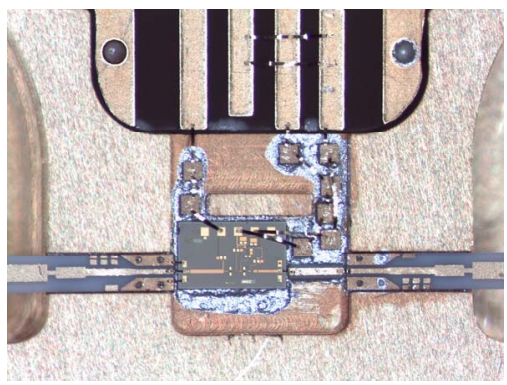

Fig. 5. Waveguide housing with assembled MMIC and thin-film probes to waveguides. Bias is provided with a PCB that includes capacitors and resistors for bias stabilization of the amplifier. 
The amplifiers were cooled to under $20 \mathrm{~K}$ ambient temperature, and noise temperature and gain characterized with hot/cold Y-factor tests. An isolator and second LNA were used in the cryostat to reduce the back-end contribution in the measurements. The back-end also included a room temperature WR-10 second harmonic mixer that contributed to the gain ripple at the low end of the frequency band. The test results in Fig. 6 demonstrate the flat, slightly above $20 \mathrm{~K}$ average noise temperature of the LNA over the full $67-90 \mathrm{GHz}$ frequency band and some frequency points where the noise temperature is less than $20 \mathrm{~K}$. These results are the lowest reported and indicate over $10 \mathrm{~K}$ improvement in the average noise temperature compared to the MIC amplifiers designed for the same band [11]. The optimum bias for lowest noise was about a factor of three to four lower in cryogenic testing at $20 \mathrm{~K}$ than at room temperature. The reduction in minimum noise temperature was a factor of seven to ten from room temperature to $20 \mathrm{~K}$ ambient. The amplifier was biased to $\mathrm{Id}=5 \mathrm{~mA}$ total current for the two stages, which corresponds to $42 \mathrm{~mA} / \mathrm{mm}$ current density on the transistors.

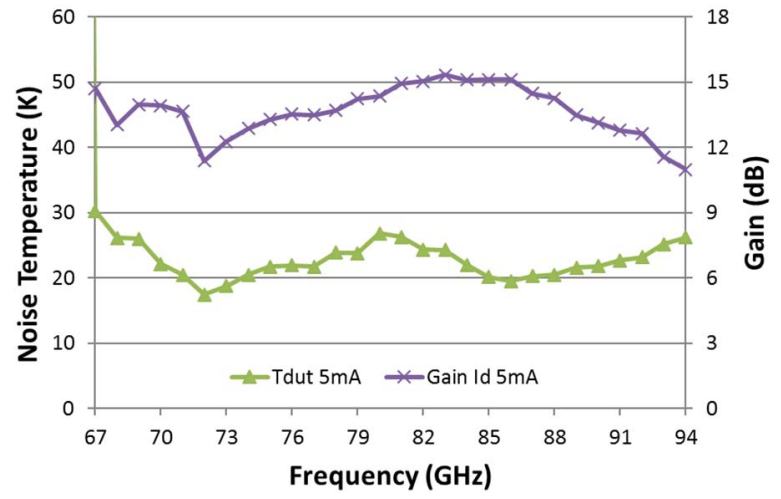

Fig. 6. Measured gain and noise temperature of the packaged 2-stage LNA at $20 \mathrm{~K}$ ambient temperature. The LNA was biased to $5 \mathrm{~mA}$ of current that corresponds to $42 \mathrm{~mA} / \mathrm{mm}$ of current density.

The results for 3-stage amplifier are shown in Fig. 7. It had minimum noise temperature of $29 \mathrm{~K}$ and average noise temperature of $34 \mathrm{~K}$ over the frequency band. The gain of the amplifier is $25 \mathrm{~dB}$, so it is suitable for use as the second stage for an amplifier module, or as the only amplifier in front of a mixer. These results were achieved with a total drain current of $10 \mathrm{~mA}$ and voltage of $0.87 \mathrm{~V}$. If the drain current is increased to $15 \mathrm{~mA}$, the noise temperature increases by $3 \mathrm{~K}$ and gain will increase by $1.5 \mathrm{~dB}$. These current values correspond to $56 \mathrm{~mA} / \mathrm{mm}$ and $83 \mathrm{~mA} / \mathrm{mm}$ current densities on the transistors.

Room temperature data for the two-stage and three-stage amplifiers are in Fig. 8. and Fig. 9. As was discussed previously, the drain current of the transistors needs to be increased for optimum room temperature operation. At room temperature the three stage design had a noise temperature of $220 \mathrm{~K}$ $(\mathrm{NF}=2.4 \mathrm{~dB})$ and $\mathrm{G}=23 \mathrm{~dB}$ of gain. These results were achieved at drain current of $\mathrm{I}_{\mathrm{d}}=30 \mathrm{~mA}$, corresponding to current density of $167 \mathrm{~mA} / \mathrm{mm}$ on the transistors.

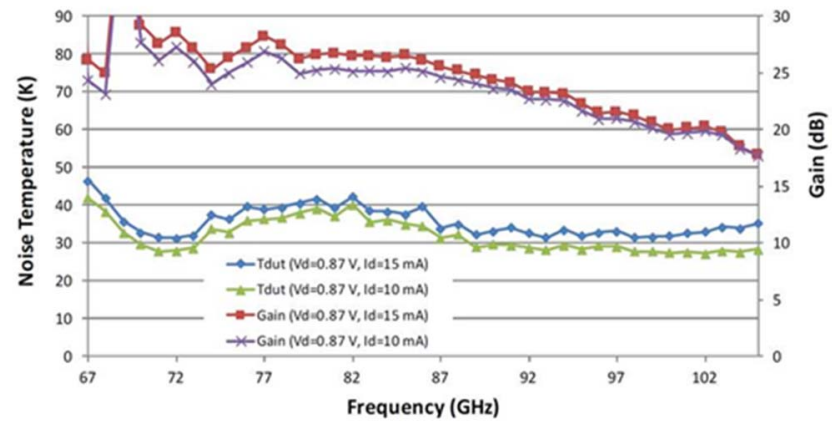

Fig. 7. Measured gain and noise temperature of packaged 3-stage LNA at $20 \mathrm{~K}$ ambient temperature. With total current of $10 \mathrm{~mA}$ the minimum noise temperature of the LNA is $29 \mathrm{~K}$.

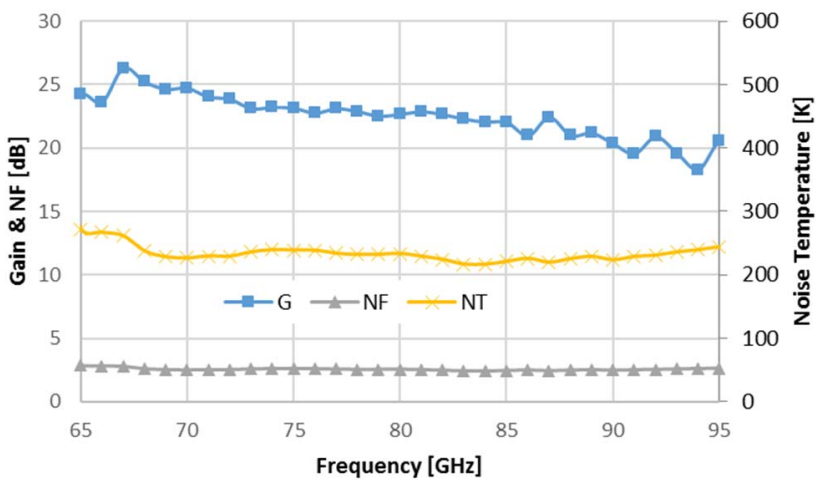

Fig. 8. Room temperature data for the three-stage LNA. The LNA is biased to $\mathrm{Vd}=1.1 \mathrm{~V}$ and $\mathrm{Id}=30 \mathrm{~mA}$. Minimum noise figure is about $2.4 \mathrm{~dB}$ and noise temperature $220 \mathrm{~K}$.

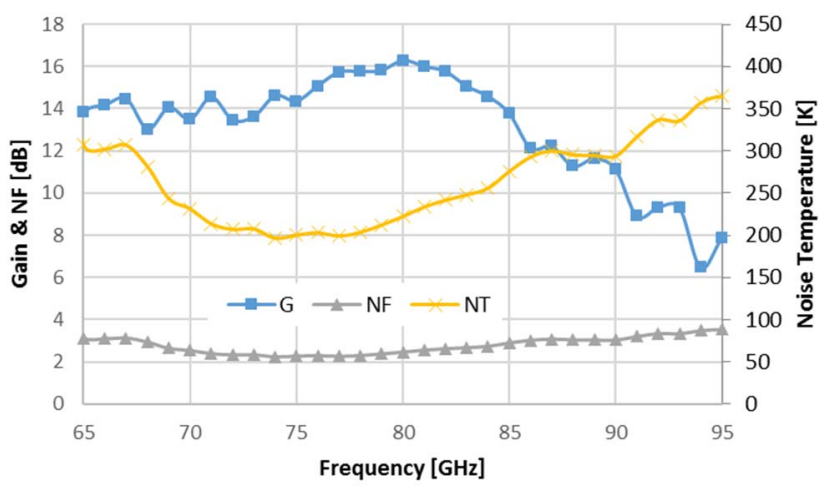

Fig. 9. Room temperature data for the two-stage LNA. The LNA is biased to $\mathrm{Vd}=1.1 \mathrm{~V}$ and $\mathrm{Id}=20 \mathrm{~mA}$ which corresponds to $167 \mathrm{~mA} / \mathrm{mm}$ current density. Noise temperature is $200 \mathrm{~K}$ and noise figure is $2.3 \mathrm{~dB}$

The two-stage amplifier results in Fig. 9. were measured at the same current density of $167 \mathrm{~mA} / \mathrm{mm}$. The drain current was $\mathrm{Id}=20 \mathrm{~mA}$ and the drain voltage $\mathrm{Vd}=1.1 \mathrm{~V}$ when the gain was $12 . .16 \mathrm{~dB}$ across the frequency band and NT $=200 \mathrm{~K}$ at mini- 
mum point $(\mathrm{NF}=2.3 \mathrm{~dB})$. The lower gain of the two-stage amplifier may have caused increase in the room temperature noise because of the losses in the microstrip lines in the housing at the output side of the amplifier (see Fig. 5.).

\section{SENTINEL-6 HRMR}

These LNAs will provide low noise for the HRMR on Sentinel-6 [5] that is not cryogenically cooled. The HRMR has internal calibration and stabilization with noise diodes and Dicke-switch. Even with the added losses from these circuits the receivers achieve $\mathrm{NT}=700 \mathrm{~K}$. With $\mathrm{BW}=10 \mathrm{GHz}$ the expected sensitivity is $\Delta \mathrm{T} / \mathrm{T}=2 \mathrm{e}-5$ (assuming $50 \%$ of the integration time is used for Dicke-switch reference load measurement and integration time is $\tau=1 \mathrm{~s}$ ). Sentinel- 6 has an orbit speed of $7 \mathrm{~km} / \mathrm{s}$, so in practice there radiometers will only be able to integrate for $\tau=0.25 \mathrm{~s}$. Thus the expected $\Delta \mathrm{T}=0.03 \mathrm{~K}$, well below the specified $0.2 \mathrm{~K}$. At longer integration times the $1 / \mathrm{f}$ noise of the amplifiers will restrict the improvement in sensitivity because of the non-gaussian nature of the $1 / \mathrm{f}$-noise. The Dicke-switching will remove this effect by allowing comparison to stable reference at a rate that is faster than the $1 / \mathrm{f}$-noise effects. Fig. 10. shows the normalized spectral density of the HRMR $90 \mathrm{GHz}$ receiver output when it is not Dicke-switched. The required Dicke-switch rate is $100 \mathrm{~Hz}$ or higher, because at that frequency the spectral density of $1 / \mathrm{f}$ noise will be equal to white noise normalized spectral density of $1 \mathrm{e}-5 \mathrm{~V} / \mathrm{V} / \mathrm{Hz}$ for this radiometer with $\mathrm{BW}=10 \mathrm{GHz}$.

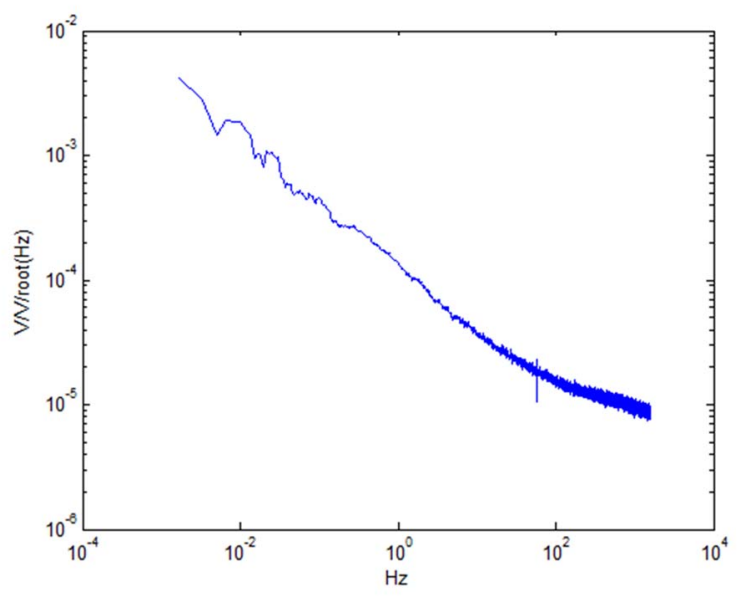

Fig. 10. Normalized spectral density at the output of the HRMR receiver. The $1 / \mathrm{f}$ noise is at $2 \mathrm{e}-4$ level at $1 \mathrm{~Hz}$ and reduces to below white noise level at above $100 \mathrm{~Hz}$.

\section{CONCLUSION}

This LNA development has demonstrated state-of-the-art results from the design of $67-90 \mathrm{GHz}$ LNAs with a high yield InP LNA process. Diced MMICs were screened in a cryogenic probe station before selecting the best performing LNAs. This technique yields excellent results for receiver arrays where hundreds of cryogenic receivers have to be built with a reasonable cost. The lowest noise MMIC LNAs can be preselected and populated as the first stages in the receiver modules. The developed LNAs enable low noise amplification for HRMR instrument on Sentinel-6. With faster than $100 \mathrm{~Hz}$ Dicke-switching rate the receivers will provide $\Delta \mathrm{T}=0.03 \mathrm{~K}$ sensitivity.

\section{ACKNOWLEDGMENT}

We would like to acknowledge I. Ramos, S. Padmanabhan, A. Tanner and T. Gaier for the measurement and analysis of the HRMR radiometer data. The research was carried out at the Jet Propulsion Laboratory, California Institute of Technology, under a contract with the National Aeronautics and Space Administration. Research was partially funded from National Radio Astronomy Observatory (NRAO) ALMA Development.

\section{REFERENCES}

[1] E. W. Bryerton, X. B. Mei, Y. Kim, W. Deal, et. al. "A W-Band LowNoise Amplifier with 22K Noise Temperature," in 2009 IEEE MTT-S Int. Microw. Symp. Dig., Boston, MA, USA, 7.-9. 2009. pp. $681-684$.

[2] L. Samoska, M. Varonen, R. Reeves, K. Cleary, R. et. al. "W-Band Cryogenic InP MMIC LNAs with Noise Below 30K," in 2012 IEEE MTT-S Int. Microw. Symp. Dig., Montreal, QC, Canada, 17.-22. June, 2012. pp. $1-4$

[3] M. Varonen, R. Reeves, P. Kangaslahti, L. Samoska, et.al. "A 75-116GHz LNA with 23-K Noise Temperature at $108 \mathrm{GHz}$," In 2013 IEEE MTT-S Int. Microw. Symp. Dig., Seattle, WA, USA, 2.-7. June, 2013. pp. 1-4.

[4] M. Kotiranta, S. Turk, F. Schafer, A. Leuther, et.al. " Cryogenic 50-nm mHEMT MMIC LNA for 67-116 GHz with $34 \mathrm{~K}$ Noise Temperature," in 2016 Global Symp. on Millim. Waves (GSMM) \& ESA Workshop on Millimetre-Wave Tech. and Appl. 6.-8. June, 2016. Espoo, Finland.

[5] J. Kloosterman, S. Brown, T. Koch, N. Vo, et.al.,"The Advanced Microwave Radiometer - Climate Quality (AMR-C) Instrument for Sentinel-6," in 2017 Int. Space Terahertz Tech. Symp. Dig., Cologne, Germany, March 2017.

[6] R. Lai, X. B. Mei, W. R. Deal, W. Yoshida, et.al. "Sub $50 \mathrm{~nm} \mathrm{InP}$ HEMT Device with Fmax Greater than $1 \mathrm{THz}$," Proc. IEEE Electron Devices Meeting, Wash., D.C., USA, 10.-12. Dec. 2007, pp. 609-611.

[7] P. M. Smith, M. Ashman, D. Xul, X. Yang, et.al. "A 50nm MHEMT Millimeter-Wave MMIC LNA with Wideband Noise and Gain Performance," in 2014 IEEE MTT-S Int. Microw. Symp. Dig., Tampa Bay, Fl, USA, 1.-6. June, 2014., pp. 1-4.

[8] M. Varonen, R. Reeves, P. Kangaslahti, L. Samoska, J.et.al. "An MMIC Low-Noise Amplifier Design Technique", IEEE Trans. on Microw. Theory and Techn., Vol. 64, Is. 3, 2016.

[9] R. Reeves, K. Cleary, R. Gawande, J. Kooi, et.al. "Cryogenic Probing of mm-Wave MMIC LNAs for Large Focal-Plane Arrays in RadioAstronomy", Proc. Europ. Microw. Conf., Rome, Italy, 6.-9. Oct., 2014,. pp. $1524-1527$.

[10] D. Russell, K. Cleary, and R. Reeves, "Cryogenic probe station for onwafer characterization of electrical devices", Rev. Sci. Instrum. 83, 044703, 2012.

[11] K.S. Saini. "ALMA Band 2 Prototype Project" in ALMA Band 2/2+3 Workshop 5, May 23rd-24th, 2016, Gothenburg, Sweden. Available: enhttps://www.chalmers.se/en/centres/GoCAS/Events/ALMABands2and3-Workshop-2016/Documents/Saini - Overview and Status of the ALMA Band 2 Prototype Project.pdf 